\title{
CONTROLE DE TIRIRICA (Cyperus rotundus) NA CULTURA DA CANA-DE-AÇÚCAR, COM O HERBICIDA IMAZAPIC ISOLADO OU EM MISTURA COM PENDIMETHALIN
}

\author{
Aimar F. F. Pedrinho Junior' ${ }^{1}$ Gustavo Martini' e Júlio C. Durigan² \\ 'Graduando em agronomia. FCAV/UNESP. Jaboticabal. SP 14870-000 \\ ${ }^{2}$ Professor Titular. Departamento de Fitossanidade, FACV/UNESP
}

\begin{abstract}
RESUMO
Visando avaliar nova alternativa para o controle químico da tiririca (Cyperus rotundus) na cultura da cana-de-açúcar (Saccharum spp.), foi conduzido um experimento, em campo, na Usina São Carlos, Jaboticabal-SP, com o herbicida imazapic. aplicado isolado e em mistura no tanque com pendimethalin, em pré-emergência. O mesmo também foi testado. adicionado de surfatante, em pós emergência das plantas cultivada e daninha. Os tratamentos testados foram: imazapic (73,5 . 98.0 , $122,5$ e $147,0 \mathrm{~g} / \mathrm{ha})$ e imazapic+pendimethalin $(50+1000,60+1200$ e $70+1400 \mathrm{~g} / \mathrm{ha})$, em pré-emergência. Em pós-emergência, o imazapic $(73,5,98,0,125,0$ e $147,0 \mathrm{~g} / \mathrm{ha})$ foi adicionado de surfatante a $0,15 \%$ do produto comercial para o volume de calda. O delineamento experimental utilizado foi o de blocos ao acaso, com quatro repetições. Para a aplicação foi utilizado um pulverizador costal, à pressão constante de $2,8 \mathrm{~kg} / \mathrm{cm}^{2}$ (mantida por ar comprimido) bicos de jato plano $\mathrm{e}$ deposição descontínua (tipo "leque") XR 11002. O consumo de calda foi equivalente a $250 \mathrm{l} / \mathrm{ha}$. A variedade de cana-deaçúcar utilizada foi a RB 855536. A maior dose de imazapic ( $147,0 \mathrm{~g} / \mathrm{ha}$ ) proporcionou, em pré emergência, os melhores resultados, com $96 \%$ de controle, aos 27 dias após o tratamento (DAA). Manteve boa percentagem (72\%) de controle até 91 DAA. As misturas de imazapic + pendimethalin não foram eficazes para o controle da tiririca. O herbicida imazapic, aplicado em pós-emergência, apresentou fitotoxidade irreversível nas plantas de cana-de-açúcar (var. RB 855536) e controlou aproximadamente $85 \%$ das plantas de tiririca.
\end{abstract}

Palavras-chave: controle químico, mistura em tanque, planta daninha.

\section{ABSTRACT \\ Purple nutsedge (Cyperus rotundus L.) control in sugar-cane crop with imazapic herbi- cide, isolated or in mixture whith pendimethalin}

The major problem with the sugar cane crop is weed competition, especially purple nutsedge which is a very agressive plant. The trial was carried out at Usina São Carlos (a sugar mill), Jaboticabal-SP, during the summer period (1999/2000) aiming to study the effect of imazapic herbicide on purple nutsedge control, sprayed at pre and postemergence, alone or in mixture with pendimethalin. The experimental design was randomized blocks with thirteen treatments and four replications. The treatments were: controls (with and without weeds), imazapic herbicide $(73.5,98.0,122.5$ and $147.0 \mathrm{~g} / \mathrm{ha}$ ), imazapic + pendimethalin $(50+1000,60+1200,70+1400 \mathrm{~g} / \mathrm{ha})$, in preemergence application. Postemergence treatments were imazapic, at the same doses, with surfactant $0.15 \% \mathrm{v} / \mathrm{v}$. The herbicide was applied with a manual sprayer, with a constant pressure of $2,8 \mathrm{~kg} / \mathrm{cm}^{2}$ and jet nozzles with descontinuous deposition (XR11002), using the equivalent of $250 \mathrm{l} / \mathrm{ha}$ of the herbicide solution. The sugar cane variety used was RB 855536 . The best pre-emergence weed control $(96 \%)$ was observed with 147 
g/ha of imazapic at 27 days after application. The postemergence applications cannot be used because they caused high toxicity in the culture. The herbicide mixture (imazapic+pendimethalin) showed low efficacy for all tested treatments.

Key words: chemical control, tank mixture, weed.

\section{INTRODUÇÃO}

A cana-de-açúcar é uma das culturas mais importantes do Brasil, possibilitando a produção, em grande escala, do açúcar e do álcool (anidro e hidratado).

O processo de globalização, com abertura de novos mercados, e a redução das reservas mundiais de combustíveis fósseis, fizeram com que a comercialização destes produtos derivados da cana-de-açúcar tomasse novo impulso. Um dos pré requisitos para que isso ocorra é a competitividade do produto final, o que implica na redução dos custos de produção, na melhoria da qualidade e redução da contaminação ambiental pelo processo produtivo.

No processo produtivo da cana-de-açúcar, um dos pontos mais críticos é a interferência negativa imposta pelas plantas daninhas que infestam as áreas cultivadas. Essas plantas podem competir por recursos limitados do meio, liberar substâncias alelopáticas que inibem a brotação da cana-de-açúcar, hospedar pragas e doenças comuns à cultura ou, ainda, interferir no rendimento da colheita. Estes componentes de interferência negativa podem causar reduções na quantidade e qualidade da cana-de-açúcar colhida, além de diminuir o número de cortes economicamente viáveis (Kuva, 1999).

A tiririca é uma das principais plantas daninhas de áreas agricolas em todo o mundo e sua interferência têm causado reduções quanti e qualitativas na produção, principalmente nas regiões tropical e subtropical (Holm et al., 1977).

$\mathrm{O}$ número de tubérculos de tiririca por $\mathrm{m}^{2}$ influencia na emissão de brotos da cana-de-açúcar, pois, quanto maior , menor a brotação (Bacchi et al, 1984). A biomassa das plantas de tiririca diminui com o aumento do tempo de convivência com a cana-de-açúcar, mas a convivência com a planta daninha também ocasiona cerca de $20 \%$ de perda para a cultura, quando comparada à produtividade de área totalmente livre de infestações (Kuva, 1999).

Poucas são as alternativas para o controle químico da tiririca, com seletividade para as plantas de cana-de-açúcar. Os dois herbicidas com os melhores desempenhos para isso são o sulfentrazone, com aplicação em pré-emergência, e o halosulfuron, em pós-emergência.

O herbicida imazapic é recomendado e registrado para as culturas do amendoim e da soja, proporcionando excelente controle de plantas daninhas e produtividade plena, mesmo causando injúrias às plantas na fase inicial do ciclo. $\mathrm{Na}$ cultura da cana-de-açúcar o uso é limitado aos testes e pouco se conhece a respeito dos sintomas de intoxicação, necessitando-se mais pesquisas para demonstrar, claramente, esses efeitos.
Segundo Shaw \& Wixson (1991), na cultura da soja a aplicação de imazapic ( $70 \mathrm{~g} / \mathrm{ha}$ ) misturado com imazaquim ( $140 \mathrm{~g} / \mathrm{ha})$, em pós emergência, causou injúrias de $8 \%$ à cultura, 56 dias após o tratamento (DAT). Entretanto, Wixson \& Shaw (1991b) constataram que a mudança na forma de aplicação destes herbicidas, injuriou mais a soja quando foram realizadas no seu estágio inicial do desenvolvimento. $\mathrm{O}$ uso de imazaquim (140 g/ha), em pré-emergência, seguido de imazapic ( $35 \mathrm{~g} / \mathrm{ha})$, em pós-emergência inicial, proporcionou injúrias de até $13 \%$, aos 56 DAT.

Durigan (1993) relata que a mistura de herbicidas pode proporcionar efeitos sinérgicos, aditivos ou antagônicos à ação dos mesmos, dependendo do tipo e classe, assim como, aos tipos de plantas daninhas que se visa controlar e a cultura em que estão ocorrendo. Surfatantes e aditivos associados ao imazapic, em pós-emergência, contribuíram para o aumento da toxicidade às plantas da soja (Wixson \& Shaw, 1991a).

Para minimizar os efeitos da fitointoxicação do herbicida pode-se associá-lo a um cultivo. O imazapic ( $9 \mathrm{~g}$ / ha), em pós-emergência, seguido de cultivo. aos sete dias após a sua aplicação, proporcionou menores injúrias. (Newson \& Shaw, 1994).

Com o objetivo de buscar novas alternativas para o controle quimico da tiririca na cultura da cana-de-açúcar, conduziu-se um experimento com o herbicida imazapic, isolado e em mistura com pendimethalin, aplicado em pré-emergência da cultura e das plantas daninhas. Também testou-se o imazapic isolado e adicionado de surfatante, aplicado em pós-emergência de ambos os tipos de plantas.

\section{MATERIAL E MÉTODOS}

O experimento foi instalado e conduzido em área da Usina São Carlos, no município de Jaboticabal, SP. O clima da região, segundo a classificação de Köeppen, é do tipo Cwa (mesotérmico de inverno seco), com temperatura média no mês mais quente de $22^{\circ} \mathrm{C}$ e no mês mais frio de $18^{\circ} \mathrm{C}$.

Os dados diários de temperatura média, precipitação e umidade relativa do ar, ocorridos durante o experimento, são apresentados na Figura 1. Nela pode-se observar que as condições climáticas eram favoráveis no momento das duas aplicações. Dias antes da aplicação em pré-emergência ocorreu uma precipitação de aproximadamente $40 \mathrm{~mm}$, o que garantiu uma boa umidade do solo no momento da aplicação. A umidade relativa do ar sempre esteve acima do nível crítico e também propiciou boas condições para a absorção. 


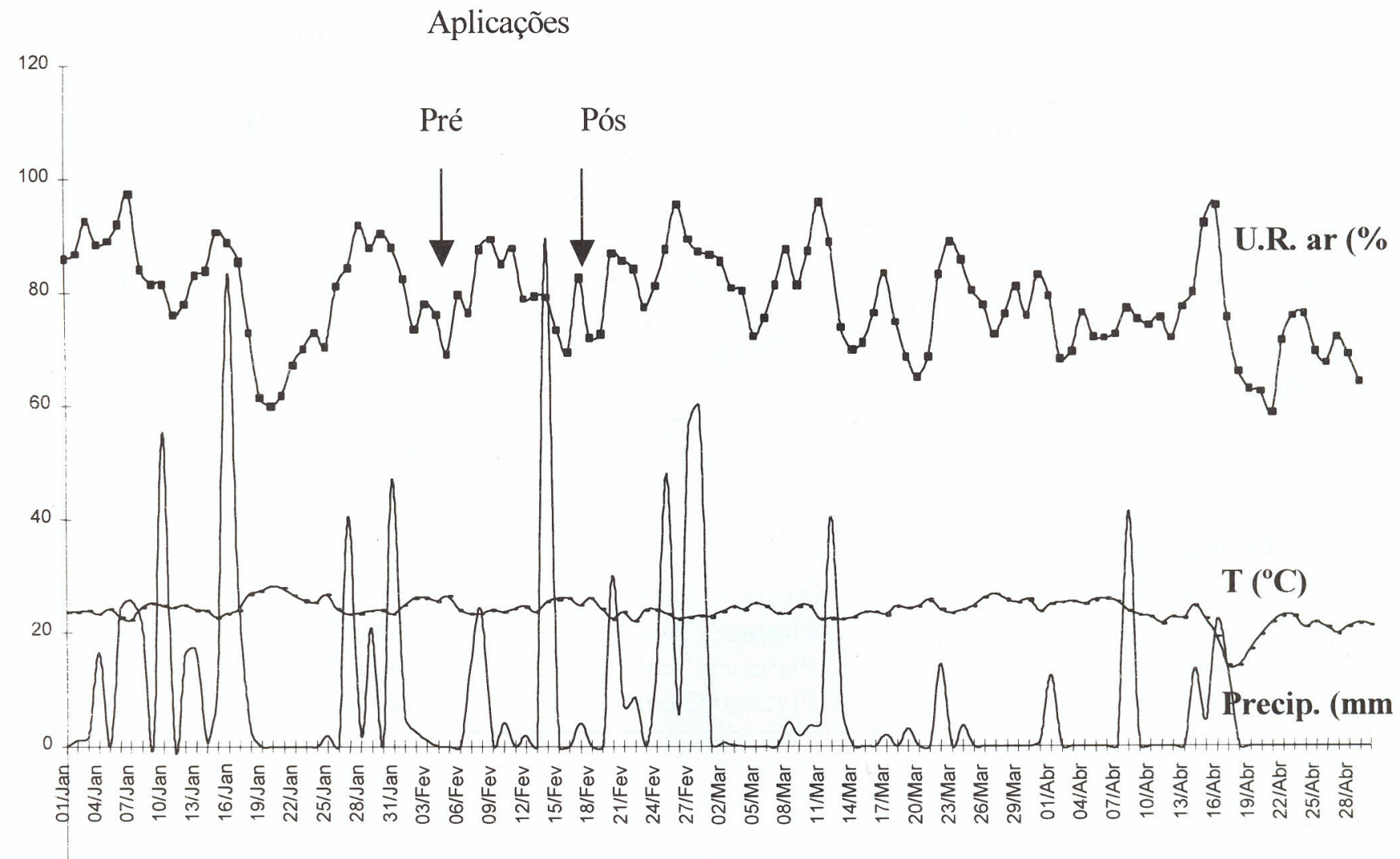

Figura 1. Temperatura média, precipitação diária e umidade relativa do ar, durante os meses de janeiro a março de 1999. (Fonte: FCAV/ UNESP).

A temperatura era superior a $25^{\circ} \mathrm{C}$ no momento de ambas as aplicações, garantindo intensa atividade metabólica das plantas que estavam começando a germinar ou que já se encontravam vegetando no local. Não ocorreu precipitação em período inferior a 48 horas após a aplicação em pós-emergência, anulando a possibilidade da chuva lavar o herbicida depositado sobre as folhas das plantas antes que fosse absorvido.

A análise granulométrica do solo apresentou os seguintes resultados, em $\mathrm{g} / \mathrm{kg}$ : argila 190, limo 50, areia fina 450 e areia grossa 310 . Foi classificado como classe textural média.

O delineamento experimental utilizado foi o de blocos ao acaso, com quatro repetições. As parcelas constaram de três linhas espaçadas de $1,4 \mathrm{~m}$ e com $8 \mathrm{~m}$ de comprimento, perfazendo área de $33,6 \mathrm{~m}^{2}$. Os herbicidas testados, com as respectivas doses, estão apresentados na Tabela 1.

O imazapic pertence ao grupo químico das imidazolinonas e apresenta-se na formulação de grânulos dispersíveis em água (GRDA), contendo $700 \mathrm{~g}$ do ingrediente ativo por quilo de produto comercial. A sua classe toxicológica ainda não está definida. O pendimethalin apresenta-se formulado como concentrado emulsionável (CE), contendo $500 \mathrm{~g}$ de ingrediente ativo por litro. Está locado na classe toxicológica II, com faixa amarela no rótulo da embalagem.
Para a aplicação utilizou-se um pulverizador costal, à pressão constante (mantida por ar comprimido) de $2,8 \mathrm{~kg} /$ $\mathrm{cm}^{2}$, munido de barra com quatro bicos de jato plano e deposição descontínua, (tipo "leque") XR 11002 e consumo de calda equivalente a $250 \mathrm{l} / \mathrm{ha}$.

$\mathrm{Na}$ aplicação em pré-emergência, dia 05/02/99, o solo encontrava-se com excelente umidade, temperatura ambiente de $38^{\circ} \mathrm{C}$, temperatura do solo (a $5 \mathrm{~cm}$ de profundidade) de $40,2^{\circ} \mathrm{C}$, nebulosidade em torno de $60 \%$ e umidade relativa do ar de $52 \%$.

A aplicação dos herbicidas, em pós-emergência, foi realizada no dia $18 / 02 / 99$, com temperatura ambiente de $33^{\circ} \mathrm{C}$, temperatura do solo (a $5 \mathrm{~cm}$ de profundidade) de $32^{\circ} \mathrm{C}$, nebulosidade em torno de $20 \%$ e umidade relativa do ar de $63 \%$. As plantas de tiririca encontravam-se com 4 a 6 folhas e as da cultura com 2 a 3 folhas, em média. A variedade de canade-açúcar utilizada foi a RB855536.

As avaliações dos herbicidas aplicados em pré-emergência foram realizadas aos 27,61 e 91 dias após a aplicação (DAA), visualmente, atribuindo-se percentagem de controle às plantas daninhas. Utilizou-se escala proposta pela SBCPD (1995) nas avaliações de intoxicação às plantas de cana. As parcelas que receberam aplicação em pós-emergência também foram avaliadas pelos mesmos métodos, aos 14, 47 e 78 DAA. Nas avaliações de controle e de fitossanidade, sempre feitas por três pessoas, as parcelas foram comparadas com as 
Aimar F. F. Pedrinho Junior et al.

Tabela 1. Herbicidas e doses aplicadas em pré e pós-emergência, para o controle de tiririca, na cultura da cana-de-açúcar. Jaboticabal, SP. 1999.

\begin{tabular}{|c|c|c|c|}
\hline \multicolumn{2}{|c|}{ Herbicida } & \multicolumn{2}{|c|}{ Dose } \\
\hline Nome comum & Nome comercial & i.a. (g/ha) & p.c. (l/ha) \\
\hline \multicolumn{4}{|l|}{ Em pré-emergência } \\
\hline $\begin{array}{l}\text { Imazapic } \\
\text { Imazapic } \\
\text { Imazapic } \\
\text { Imazapic } \\
\text { Imazapic+pendimethalin } \\
\text { Imazapic+pendimethalin } \\
\text { Imazapic+pendimethalin }\end{array}$ & $\begin{array}{c}\text { Plateau } \\
\text { Plateau } \\
\text { Plateau } \\
\text { Plateau } \\
\text { Plateau+Herbadox } \\
\text { Plateau+Herbadox } \\
\text { Plateau+Herbadox }\end{array}$ & $\begin{array}{c}73,5 \\
98,0 \\
122,5 \\
147,0 \\
50+1000 \\
60+1200 \\
70+1400\end{array}$ & $\begin{array}{c}105 \mathrm{~g} \\
140 \mathrm{~g} \\
175 \mathrm{~g} \\
210 \mathrm{~g} \\
71 \mathrm{~g}+2,0 \mathrm{l} \\
86 \mathrm{~g}+2,4 \mathrm{~g} \\
100 \mathrm{~g}+28 \mathrm{l}\end{array}$ \\
\hline \multicolumn{4}{|l|}{ Em pós-emergência } \\
\hline $\begin{array}{l}\text { Imazapic+surfatante } \\
\text { Imazapic+surfatante } \\
\text { Imazapic+surfatante } \\
\text { Imazapic+surfatante }\end{array}$ & $\begin{array}{l}\text { Plateau+Cicol } \\
\text { Plateau+Cicol } \\
\text { Plateau+Cicol } \\
\text { Plateau+Cicol }\end{array}$ & $\begin{array}{c}73,5 \\
98,0 \\
122,5 \\
147,0\end{array}$ & $\begin{array}{l}105 \mathrm{~g} \\
140 \mathrm{~g} \\
175 \mathrm{~g} \\
210 \mathrm{~g}\end{array}$ \\
\hline
\end{tabular}

(1) Cicol a $0,15 \%$ v/v na calda.

i.a. $=$ ingrediente ativo

p.c. $=$ produto comercial

testemunhas infestada e capinada.

Aos 91 (pré) e 78 (pós) DAA, foi efetuada a medição de altura das plantas até a última aurícula visível.

\section{RESULTADOS E DISCUSSÃO}

A principal planta daninha da área experimental e objetivo de controle era a tiririca, cobrindo, em média, 75\% do solo. Conforme pode ser observado na Tabela 2 , o herbicida imazapic, isolado e em pré-emergência, nas doses de 122,5 e $147,0 \mathrm{~g} / \mathrm{ha}$, proporcionava controle de 94 e $96 \%$, respectivamente, aos 27 DAA, diferindo estatisticamente dos tratamentos onde foi misturado ao pendimethalin. Nesta mesma data observa-se que, nos tratamentos onde o imazapic foi aplicado em pós-emergência, o controle, em torno de $70 \%$, não diferia entre eles. Aos 91 DAA em pré-emergência, o controle era de $64 \%$ para a dose de $122,5 \mathrm{~g} / \mathrm{ha}$ e $72 \%$ para a de $147,0 \mathrm{~g} / \mathrm{ha}$, sem diferença estatística dos demais tratamentos.

A concentração do herbicida diminuiu no solo com o tempo e novas brotações surgíram, fazendo com que os percentuais de controle atribuídos fossem menores. Aos 61 DAA apenas as duas maiores doses do herbicida imazapic, isolado e em pré-emergência, ainda mantinham controle superior a $80 \%$. Na última avaliação, aos 91 DAA, apenas na maior dose $(147,0 \mathrm{~g} / \mathrm{ha})$ o controle era superior a $70 \%$.

A mistura dos herbicidas não beneficiaram o resultado final de controle, pois a redução de dose do imazapic não foi compensada pela ação do pendimethalin colocado na mistura. As maiores doses testadas em mistura, proporcionavam controle de $66 \%$ enquanto que na maior do imazapic isolado era de $89 \%$, aos 61 DAA. Para esta planta daninha o pendimethalin não exerceu ação fitotóxica direta e nem sinergismo para a atuação do imazapic.

A ação do herbicida imazapic adicionado de surfatante, em pós-emergência, também foi efetiva e atingiu o ápice aos 48 DAA, com controle superior a $85 \%$ nas três doses maiores. Na última avaliação, apesar da redução ocorrida, os percentuais de controle mantinham-se iguais ou superiores a $70 \%$ nestes mesmos tratamentos.

Os resultados das avaliações visuais de fitotoxicidade, apresentados na Tabela 3, mostram que as doses menores de imazapic (73,5 e 98,0 g/ha), aplicadas em pré-emergência evidenciaram possibilidade de recuperação, como ocorreu no trabalho realizado por Azânia (2000).

$\mathrm{Na}$ Tabela 4 observa-se que as doses de 122,5 e 147,0 $\mathrm{g} /$ ha de imazapic, proporcionaram injúrias moderadas, com redução de altura das plantas de cana-de-açúcar, apesar de não diferirem estatisticamente dos outros tratamentos em préemergência, e requerem atenção e novos experimentos para esta e outras variedades de cana-de-açúcar, bem como para diferentes tipos de solos.

Segundo Wixson \& Shaw (1992), a aplicação de imazapic $(9,18$ e $35 \mathrm{~g} / \mathrm{ha})$ em pré-emergência, também causou até $20 \%$ de injúria inicial em diferentes culturas (soja, feijão, algodão e milho). O resultado obtido por Azânia (2000) mostra que, para a dosagem de $122,5 \mathrm{~g} /$ ha de imazapic, a fitotoxicidade aos $30 \mathrm{DAA}$ foi pequena, com notas iguais ou 
Tabela 2. Médias das percentagens de controle atribuídas nas avaliações visuais feitas para tiririca (Cyperus rotundus) na cultura da cana de açúcar, em diferentes dias após a aplicação dos herbicidas (DAA). Jaboticabal, SP. 1999.

\begin{tabular}{|c|c|c|c|c|}
\hline \multirow{3}{*}{ Tratamento } & \multirow{3}{*}{$\begin{array}{c}\text { Dose } \\
\text { (g/ha) }\end{array}$} & \multicolumn{3}{|c|}{ Cobertura do solo pela planta daninha $(\%)$} \\
\hline & & \multicolumn{3}{|c|}{ Em pré-emergência (DAA) } \\
\hline & & 27 & 61 & 91 \\
\hline Testemunha infestada & - & $0 *$ e $(70)^{* *}$ & $0 \mathrm{~g} \quad(84)$ & 0 e $\quad(78)$ \\
\hline Testemunha capinada & - & 90 a $(100)$ & 90 a $(100)$ & 90 a $(100)$ \\
\hline Imazapic & 73,5 & $63 \mathrm{~cd}(85)$ & 54 ef $(70)$ & $51 \mathrm{bcd}(60)$ \\
\hline Imazapic & 98,0 & 71 bc $(90)$ & $61 \mathrm{cde}(76)$ & $54 \mathrm{bcd}(65)$ \\
\hline Imazapic & 122,5 & 76 b (94) & 65 bcd $(81)$ & 53 bcd (64) \\
\hline Imazapic & 147,0 & $80 \mathrm{ab}(96)$ & $71 \mathrm{bc} \quad(89)$ & $58 \mathrm{~b} \quad(72)$ \\
\hline Imazapic + pendimethalin & $50+1000$ & $62 \mathrm{~cd}(78)$ & $48 \mathrm{f} \quad(55)$ & $46 \mathrm{~d} \quad(52)$ \\
\hline Imazapic+pendimethalin & $60+1200$ & $63 \mathrm{~cd}(79)$ & 52 ef $(62)$ & $47 \mathrm{~d}$ \\
\hline \multirow[t]{3}{*}{ Imazapic+pendimethalin } & $70+1400$ & $65 \mathrm{~cd}(81)$ & 55 def (66) & $48 \mathrm{~cd}(56)$ \\
\hline & & \multicolumn{3}{|c|}{ Em pós-emergência (DAA) } \\
\hline & & 14 & 48 & 78 \\
\hline${\text { Imazapic }+ \text { surfatante }^{1}}^{1}$ & 73,5 & $57 \mathrm{~d}(70)$ & 60 cde $(75)$ & $48 \mathrm{~cd} \mathrm{(55)}$ \\
\hline Imazapic+surfatante & 98,0 & $56 \mathrm{~d}(69)$ & $67 \mathrm{bc} \quad(85)$ & 57 bc $(70)$ \\
\hline Imazapic + surfatante & 122,5 & $55 \mathrm{~d}(68)$ & $68 \mathrm{bc} \quad(85)$ & $57 \mathrm{bc} \quad(70)$ \\
\hline Imazapic+surfatante & 144,0 & $60 \mathrm{~d}(75)$ & (91) & $59 \mathrm{~b}$ \\
\hline $\mathrm{F}$ & & $91,4 * *$ & $94,4 * *$ & $97,5 * *$ \\
\hline DMS & & 11,1 & 10,7 & 9,6 \\
\hline C.V. $(\%)$ & & 7,2 & 7,2 & 7,5 \\
\hline
\end{tabular}

(1) Cicol a $0,15 \% \mathrm{v} / \mathrm{v}$ na calda.

* Dados transformados em arc sen $\sqrt{\mathrm{x}}$

**Dados originais

Tabela 3. Conceitos atribuídos nas avaliações visuais de fitotoxicidade, segundo recomendações da SBCPD, para a cultura da cana-de-açúcar, em diferentes dias após a aplicação dos herbicidas (DAA). Jaboticabal, SP. 1999.

\begin{tabular}{|c|c|c|c|c|}
\hline \multirow{3}{*}{ Tratamento } & \multirow{3}{*}{$\begin{array}{c}\text { Dose } \\
(\mathrm{g} / \mathrm{ha})\end{array}$} & \multicolumn{3}{|c|}{ Época de avaliação da fitotoxicidade (DAA) } \\
\hline & & \multicolumn{3}{|c|}{ Em pré-emergência } \\
\hline & & 27 & 61 & 91 \\
\hline Testemunha in festada & - & A & A & A \\
\hline Testem unha capinada & - & A & A & $\mathrm{A}$ \\
\hline Imazapic & 73,5 & $\mathrm{~B}$ & $\mathrm{~B}$ & $\mathrm{~B}$ \\
\hline Im azapic & 98,0 & $\mathrm{~B}$ & $\mathrm{C}$ & $\mathrm{B}$ \\
\hline Im azapic & 122,5 & $\mathrm{C}$ & $\mathrm{C}$ & $\mathrm{C}$ \\
\hline Imazapic & 147,0 & $\mathrm{C}$ & $\mathrm{C}$ & $\mathrm{C}$ \\
\hline Imazapic + pendimethalin & $50+1000$ & $\mathrm{~B}$ & B & $\mathrm{B}$ \\
\hline Imazapic + pendim ethalin & $60+1200$ & $\mathrm{~B}$ & B & $\mathrm{B}$ \\
\hline \multirow[t]{3}{*}{ Imazapic + pendimethalin } & $70+1400$ & $\mathrm{~B}$ & B & $\mathrm{B}$ \\
\hline & & \multicolumn{3}{|c|}{ Em pós-emergência } \\
\hline & & 14 & 48 & 78 \\
\hline Im azapic+surfatante ${ }^{(1)}$ & 73,5 & $\mathrm{D}$ & $\mathrm{D}$ & $\mathrm{C}$ \\
\hline Im azapic+surfatante & 98,0 & $\mathrm{D}$ & $\mathrm{D}$ & $\mathrm{D}$ \\
\hline Im azapic+surfatante & 122,5 & $\mathrm{D}$ & $\mathrm{D}$ & $\mathrm{D}$ \\
\hline Imazapic+surfatante & 144,0 & $\mathrm{D}$ & $\mathrm{D}$ & $\mathrm{D}$ \\
\hline
\end{tabular}

(1) Cicol a $0,15 \% \mathrm{v} / \mathrm{v}$ na calda. 
Tabela 4. Médias de altura das plantas de cana-de-acúcar até a última aurícula visível, aos 91 e 78 dias após a aplicação (DAA), para as aplicações em pré e pós-emergência. Jaboticabal, SP. 1999.

\begin{tabular}{lcc}
\hline \multicolumn{1}{c}{ Tratamento } & $\begin{array}{c}\text { Dose } \\
\text { (g/ha) }\end{array}$ & $\begin{array}{c}\text { Altura Média } \\
(\mathbf{c m})\end{array}$ \\
\hline Em pré-emergência (91 DAA): & \\
Testemunha infestada & - & $24,9 \mathrm{~A}$ \\
Testemunha capinada & - & $25,3 \mathrm{~A}$ \\
Imazapic & 73,5 & $20,0 \mathrm{ABC}$ \\
Imazapic & 98,0 & $18,3 \mathrm{ABC}$ \\
Imazapic & 122,5 & $18,2 \mathrm{ABC}$ \\
Imazapic & 147,0 & $16,2 \mathrm{ABC}$ \\
Imazapic+pendimethalin & $50+1000$ & $19,9 \mathrm{ABC}$ \\
Imazapic+pendimethalin & $60+1200$ & $19,9 \mathrm{ABC}$ \\
Imazapic+pendimethalin & & $23,7 \mathrm{AB}$ \\
\hline Em pós-emergência (78 DAA): & \\
Imazapic+surfatante ${ }^{(1)}$ & 73,5 & $16,6 \mathrm{abc}$ \\
Imazapic+surfatante & 98,0 & $13,5 \mathrm{bc}$ \\
Imazapic+surfatante & 122,5 & $12,0 \mathrm{c}$ \\
Imazapic+surfatante & 147,0 & $13,0 \mathrm{bc}$ \\
\hline F & & $4,1 * *$ \\
DMS & & 10,8 \\
C.V. (\%) & & 23,1 \\
\hline
\end{tabular}

(1) Cicol a $0,15 \% \mathrm{v} / \mathrm{v}$ na calda.

inferiores a 2, segundo o EWRC (1964), com plena recuperação posterior.

Os tratamentos de imazapic com surfatante, em pósemergência, proporcionaram injúrias severas às plantas da cultura e grande redução no desenvolvimento. Isso pode ser explicado pelo fato do herbicida não ter boa seletividade para a variedade de cana-de-açúcar utilizada e ainda ter seu efeito potencializado pelo surfatante utilizado. Segundo Coleti \& Nunes (1982), variedades de cana-de-açúcar podem apresentar diferentes níveis de sintomas de fitotointoxicação para o mesmo herbicida, constatando-se morte de plantas em algumas e plena tolerância em outras. A variação de tolerância a herbicidas entre cultivares também foi observada em soja por Griffin et al. (1993).

Os resultados obtidos na avaliação de altura das plantas de cana-de-açúcar, aos 91 DAA em pré-emergência, e 78 DAA em pós-emergência, são apresentados na Tabela 4. A cultura mostrou sensibilidade maior ao herbicida aplicado em pós-emergência praticamente descartando-o para esse objetivo. Além da redução no desenvolvimento das plantas da cultura, observou-se arroxeamento e leve clorose das folhas.

\section{CONCLUSÕES}

A maior dose (147,0 g/ha) do herbicida imazapic isolado, aplicado em pré-emergência, proporcionou controle superior a $85 \%$ até 61 dias após a aplicação.

A mistura imazapic + pendimethalin $(50+1000$, $60+1200$ e $70+1400 \mathrm{~g} / \mathrm{ha}$ ) proporcionou um controle de apenas $60 \%$, em média, de Cyperus rotundus.

Apesar do bom controle com imazapic aplicado em pós-emergência, nas doses de 98,0,122,5 e 147,0 g/ha com surfatante a $0,15 \%$ na calda, a sua aplicação é inviável para a variedade de cana-de-açúcar RB 855536, devido aos seus fortes efeitos fitotóxicos.

\section{LITERATURA CITADA}

AZANIA, C. A. M. Seletividade de imazapic às soqueiras de cana-de-açúcar. Faculdade de Ciências Agrárias e Veterinárias -UNESP, Campus de Jaboticabal. Jaboticabal, SP. 2000. (Dissertação de Mestrado).

BACCHI, O. O. S.; ROLIN, J.C.; CHRISTOFFOLETI, P.J. Efeitos da tiririca (Cyperus rotundus L.) sobre a brotação da cana-de-açúcar. Saccharum APC, v.7, n.32, p.44 -48, 1984.

COLETI, J.T.; NUNES JR, D. Avaliação inicial da fitotoxicidade de herbicidas sobre 16 cultivares de cana-de-açúcar (Saccharum spp). In: CONGRESSO BRASILEIRO DE HERBICIDAS E ERVAS DANINHAS, VI Congreso de la Asociación Latino-Americana de Malezas, 14, Campinas, SP, Resumos... p.89, 1982.

DURIGAN, J.C. Efeitos de adjuvantes na aplicação e eficácia dos herbicidas. FUNEP, 1993. 42p. (Boletim Científico).

GRIFFIN, J.L.; REYNOLDS, D.B.; VIDRINE, P.R.; BRUFF, S.A. Soybean (Glicine max) tolerance and sicklepod (Cassia obtusifolia) control with AC 263, 222. Weed Technology., v.7, p.331-336, 1993.

HOLM, L.G.; PLUNKNETT, D.L.; PANCHO,J.V.; HERBERGER, J.P. The wold's worst weeds. Honolulu: University Press of Hawaii, 1977. 607p.

KUVA, M.A. Efeito de período de controle e de convivência das plantas daninhas na cultura da cana-deaçúcar (Saccharum sp) no Estado de São Paulo. Escola Superior de Agricultura Luiz de Queiroz. Piracicaba, SP. 1999. 74p. (Dissertação de Mestrado).

NEWSON, L.J.; SHAW, D.R. Influence of cultivation timing on weed control in soybean (Glicine max) with AC 263,222. Weed Technology, v.8, p. 760-765, 1994.

SHAW, D.R.; WIXSON, M.B. Postemergence combinations of imazaquim or imazethapyr with AC 263,222 for 
weed control in soybean (Glycine max). Weed Science, v.39, p.644 - $649,1991$.

SOCIEDADE BRASILEIRA DA CIÊNCIA DAS PLANTAS DANINHAS. Procedimentos para instalação, avaliação e análise de experimentos com herbicidas. Londrina: SBCPD, 1995. 42p.

WIXSON, M.B.; SHAW, D.R. Effects of adjuvants on weed control and soybean (Glicine $\max$ ) tolerance with $\mathrm{AC}$
263,222. Weed Technology, v.5, p.817-822, $1991 \mathrm{a}$.

WIXSON, M.B.; SHAW, D.R. Use of AC 263,222 for sicklepod (Cassia obtusifolia) control in soybean (Glicine max). Weed Technology, v.5, p.434-438, $1991 \mathrm{~b}$.

WIXSON, M.B.; SHAW, D.R. Effects of soil-applied AC 263,222 on crops rotated with soybean (Glicine max). Weed Technology, v.6, p.276-279, 1992. 
Revista Brasileira de Herbicidas, v.2, n.1, 2001. 